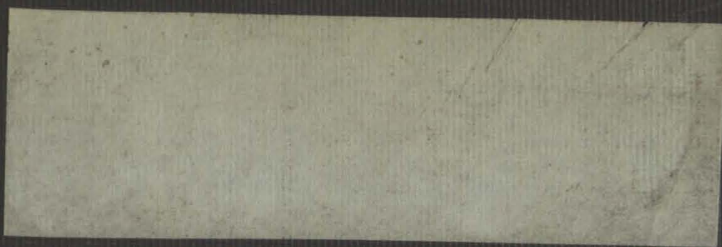

UM Libraries Depository 

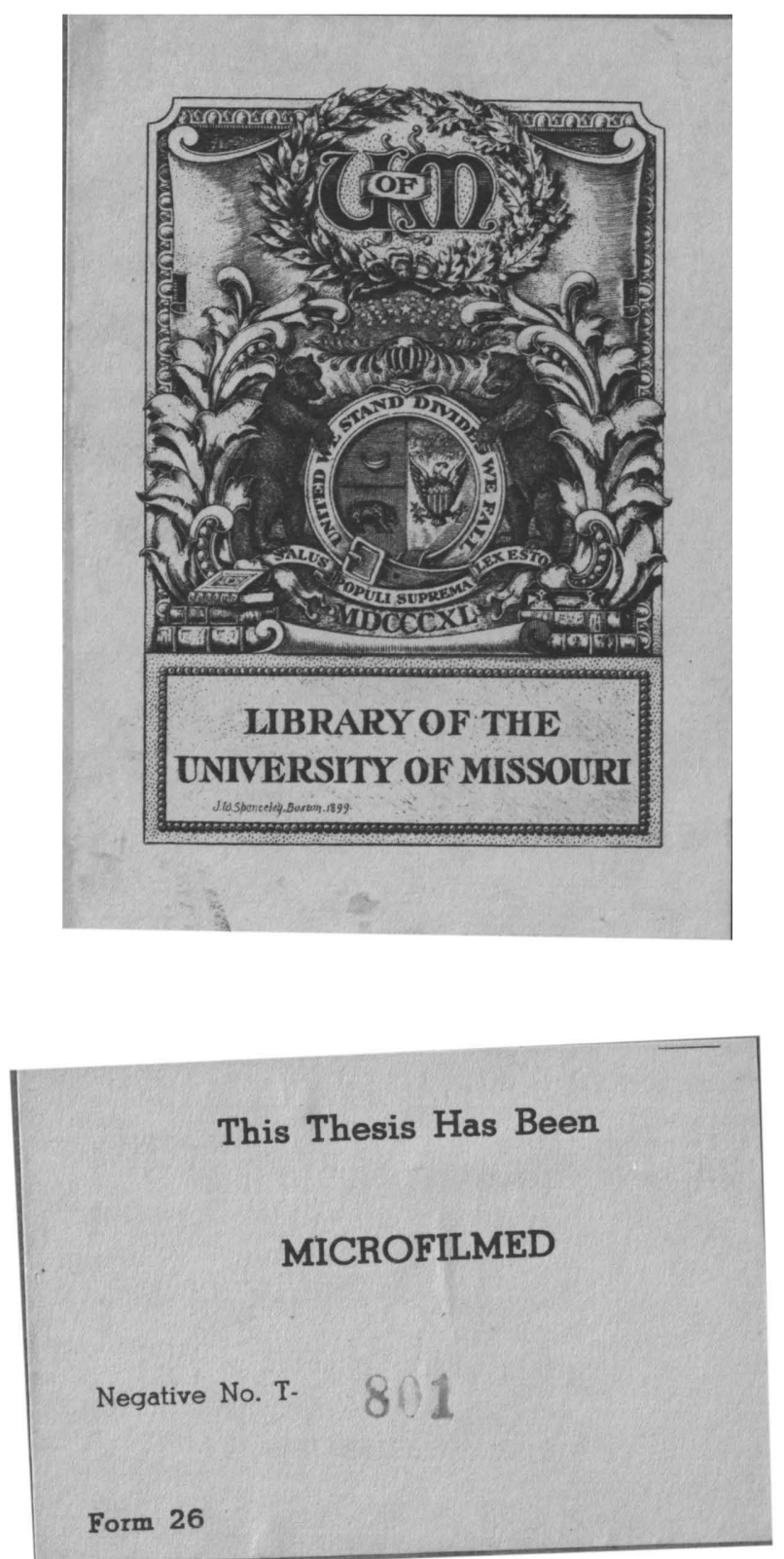




\section{CYTOLOGICAL STUDIES ON THE ZYGOSPORES \\ OF \\ SPORODINIA GRANDIS}

by

Mary Lucille Keene, B.S. in Ed., A.B.

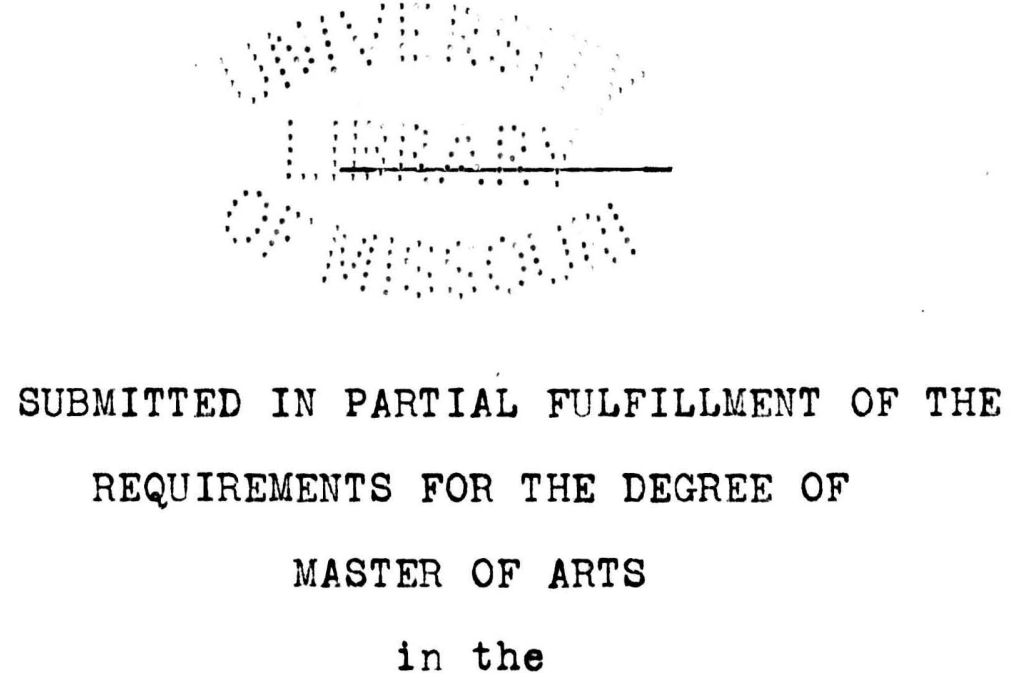

GRADUATE SCHOOL

of the

UNIVERSITY OF MISSOURI

1912 

In recent years much interest has been aroused in the cytology of the lower fungi, and considerable work has been done upon these forms. While various members of different groups have been studied, the most definite results have been obtained in the Entomopthorineae and the Oomycetes. Although several of the Mucorineae have been worked out, more or less thoroughly, the results obtained by the different investigators, have been, to some extent, contradictory. As a result some uncertainty still exists with regard to the internal processes connected with sexual fusions. Further investigation seems, therefore, to be desirable, and the present study was undertaken for the purpose of adding something, if possible, to our knowledge of these internal activities.

Inasmuch as Sporodinia grandis is an abundant and widely distributed form among the Mucorineae, it has furnished since earliest times a particularly favorable form for investigation. It is easily obtaine since it grows, parasitically, on many of the higher fungi, e.g. the Agarics; moreover, it can be obtained readily in cultures on various media. Here the zygospores are $111009^{59}$ 

produced abundantly. The sexual hyphae arise from the same mycelium, and in many cases from the same dichotomy, and the zygospores are borne on aerial branches. This habit of growth makes it possible to obtain all stages of copulation. HISTORICAL •

As early as 1820, Ehrenberg(1) described the process of conjugation in a fungus that he termed Syzygites; one of the Mucorineae. Later this form was given the name of Sporodinia grandis. It is evident, therefore, that the phenomenon of fusion in the simple forms was recognized and described very rearly. In 1864, A. de Bary described the formation of the zygespore. "The true fruiting branches are branched dichotomously repeatedly. On every two of the hyphae are formed pear-shaped enlargements. The suspensors grow towards eachother, meet, and the ends are cut off by partitions, and in this manner the gametes are formed. The membranes on the contiguous surfaces are dissolved away, the contents fuse and so results the zygospore. The same is encircled by three membranes, the outermost of which is dark brown, warty, and cutinized,formed by the membranes of the 

copulating gametes. The two inner ones belong to the zygospore itself. The outer of these two, or the middle of the three,is more or less thick, transparent, and stratified, while the innermost consists only of a thin membrane." (z)

Through the work of Brefeld(3) in 1872, and also that of Van Tieghem and Le Monnier (4), 1873-75, the Mucorineae are well known from a morphological point of view. These authors, however, go no further into the internal structure of these forms than to say that the protoplasm contains many small nuclei.

In 1894-95, Dangeard and Leger, (5), in investigations on the structure and sexuality of the Nucorinea using Sporodinia Exandis as the form for study, undertook to work out the internal structures of developing zygospores. They were the first to use the more modern methods of histology in order to gain some knowledge of the internal activities carried on in these forms. They described the young zygospore as a cask-like structure with an outer cutinized envelope, which had characteristic papillae-like projections. Internally, the protoplasm is dense and homogeneous containing many nuclei. The separating walls are dissolved, leaving the gametes in free communication. The lateral walls show 

a central brown spot and a quantity of small openings of various sizes, which favor interchange with the suspensors that contain nuclei and protoplasm. The protoplasm is reticulate, with meshes towards the surface. Nuclei of two kinds appear, some of which are two or three times the size of the ordinary nuclei, and have a dense, rounded, red-staining nucleolus. The smaller nuclei are reduced to nucleoli that stain $r e d$.

At an older stage, when oil begins to accumulate in the center of the zygospore, the nuclei are still evident in the protoplasm. No difference in the size of the nuclei is noted at this time. In the mature zygospore, the oil globule is very large and the protoplasm has become reduced to a parietal layer, dense at certain points and vacuolated over the rest. Mucorine crystals are present at this stage.

Maurice Leger (6) in 1895 made a more extensive study. He undertook a rather thorough investigation of Sporodinia grandis and, on the whole, described many of the structures that more recent work has brought out. This plece of research and its fesults has never been generally accepted as entirely satisfactory. This 

was due probably to the fact that Leger in this paper de= scribed many curfous and unusual structures. The histological methods of 1895 were far less accurate than they are today, and it is likely that this would explain many of the difficulties that Leger encountered. Even with the aid of modern technique, the problem has proved a difficult one, and Leger's misinterpretations of the structures is readily understood.

Following is a brief review of the work done by Leger. The pyriform swellings that fuse are identical and contain many nuclei. He emphasizes the absolute isogamy of the gametes, and the inability to differentiate between the two, either externally or internally. His account varies little from that given earlier by Dangeard and himself, up to the point where the zygospore is formed. He lays some stress on the formation of a peculiar structure in the walls adjacent to the suspensors, and interprets it as a canal. He also describes the disintegration of some nuclei. The protoplasm becomes vacuolate and appears as a sponge filled with oil. The nuclei disappear at this time, and at the same time there become evident certain deep-otaining bodies, 

difficult of interpretation. They arise as small spherical bodies, towards the extremities of the zygospore. He questions their origin but concludes that they arise, either from the large nuclei, or from selected protoplasm. He was unable to detect any details of structure. These spheres are isolated at first, but ultimately arrange themselves in a spherical zone around an oil mass. He describes these masses as varying in size in different zygospores, but being uniform within the same one. These small spheres Leger calls"spheres embryagenes", and the larger ones realting from their fusion, he calls "spheres: embryonnaires". He justifies the terms on the basis of his interpretation of their ultimate function, namely, that they function directly in the reprodhction of the plant when the zygospores germinate.
\end{abstract}

In germination, the spheres enlarge and fuse, al though he can discern no definite organization in them. Characteristic nuclei appear again; they divide and pass out into the hypha. The oil is absorbed little by little. In summarizing, Leger emphasizes the importance of the 

"embryo spheres" saying, "These bodies constitute in uniting a sort of embryo which develops into a new plant at the time of germination: the embryo is nourished by the reserve substances contained intthe egg when it is cut off."

In 1901, Eduard Gruber(7) undertook the further investigation of this form. He failed to find the structures described by Leger. He finds neither fusion nor disintegration of the nuclei. He points out that his work has at least disqualified that of Leger, but as he offers nothing new it is practically without value. He suggests that here we have to do with processes similar to those displayed in the oomycetes.

Mottier (8) in 1904 in a short review, summarizes the investigations to date. He refers to the sexual act as wager regards it, as being the union of the nuclei to form the embryo sphere. He suggests that because of what is now known concerning the sexual union of multi-nucleate cells that it is probable that a union of the nuclei in pairs obtains also in Sporodinia. Dangeard (9)in a short paper in 1906, describes nuclear fusions in the zygospores of Mucor fragilis and Sporodinia 

grandis, and points out that copulation occurs progressively. The nuclear fusions present the ordinary conditions of fusion of the karyoplasm and ultimately of the nucleoli.This results in the presence in the zygospore, of a large number of nuclei doubled by copulation. Dangeard here points out the fact that the two fusing structures are not to be regarded as gametes but gametangia, and the nuclei are then to be regarded as gametes, being "sexual energids" further undifferentiated morphologicallys In another paper in 1906, Dangeard (10) takes up the structure and development of the Mucorineae. His chief studies were made on the form Mucor fragilis because it contains a small number of nuclei. One of the discouraging factors in the study of Sporodinia has been the fact that the nuclei are so minute and numerou that it requires extremely high powers of magnification for their study. But, on the whole, Dangeard has investigated Sporodinia more closely than any previous worker and his results are important.

It was omly after describing the nuclear fusions in Mucor fragilis that Dangeard was able to return to Sporodinia 

and describe the fusions there. The account up to the place where nuclear fusions take place varies but little from that given in previous reviews. Dangeard describes the nuclear fusions as occuring after the endospore has been established, and as the protoplasmic portions become vacuolated.

In the mature zygospore, the vacuolate structure gives way to an alveolar structure in which is contained the oil and other reserve substances. The zygospores contain chromatic bodies which are made up of a dense homogeneous substance which stains red with the triple stain. These corpuscles are usually very large and two or three appear in a section. Their position is not constant and they vary in size within the same zygospore. Their mass is without structure and they are contained on the inside of a vacuole. Dangeard likens them to the coenocentra described by Stevens in Albugo. He describes similar structure as occuring in the suspensors and also in the mycelium. In the older zygospores, Dangeard suggests that the large corpuscles numbering 10-20, are merely mucorine crystals which have undergone reconstruction. They have increased in volume and lost their 

crystalline nature. Dangeard then goes into an account of the formation of the membranes of the zygospore which does not concern the immediate problem in hand.

Lendner (11) in 1908, undertook the histological study of the zygospores of Sporodinia. He did not describe the multiple nuclear fusions and nuclear degeneration found by Dangeard, but found a fusion between two large nuclei, with two chromosomes respectively. Each nucleus is said to originate in one of the two copulating structures. In 1911, Moreau (12) investigated several forms; Sporodinie grandis, a fucor, and Zygorhynchus. In Sporodinia and Mucor, he merely corroberates the work of Dangeard. In Zygorhynchus, he went furthior into, the details and found varying structures. All the nuclei, in this form, are said th disintegrate excopt four, which fuse in pairs leaving two nuclei in the mature zygospore.

In January 1912, Miss McCormick(13) published a preliminary notice on some investigations on the closely related genus Rhizopus nigricans. She described many nuclei in the 

younger stages. All of these nuclei disintegrate except two. These, in connection with coenocentra, increase greatly in size without any nuclear fusions occuring. According to Miss McCormick these coenocentra were identified by Professor Stevens as identical with the coenocentra described by him in Albuge. Miss NoCormick in her work indicates that the coenocentra have their origin at the point of contact of the suspensors before the gametangia are cut off. The coenocentra persist until late, and in the matute zygospore are many nuclei of the same size as those in the mycelium. Neither fusion nor division of nuclei was observed by her. In the mature zygospore there was usually only one oil globule, while in the younger stages the oil was diffused through out in small globules.

In a general view of the literature that has been available on this problem, it seems evident that the problem is still unsettled. On the one hand Leger, Dangeard, and Moreauhold that numerous nuclel are present at all stages, and that nuclear disintegration occurs in the zygospore. Dangeard and Moreau describe 

nuclear fusions which occur between many pairs of nuclei. On the other hand, Lendner in Sporodinia, and Miss McCormick in Rhizopus find numerous nuclei in the younger stages only. At maturity all the nuclei but two disintegrate. Lendner describes fusion here, but Miss McCormick fails to find this in Rhizopus.

All investigators have encountered structures that they have described under various names and attributed different functions to them. All have admitted that their interpretation was difficult. Therefore, it has seemed advisable to make an attempt to substantiate the results of one or the other group of workers, and to explain, if possible, the nature, structure, and function of the other zygospore inclusions. 

METHODS .

The cultures used in the following experiments were obtained in October 1911; if the vicinity of Columbia, Missouri, growing on mushrooms. Isolations and cultures on bread were obtained, and great numbers of zygospores matured from the first. Various stages of the material were collected and fixed. Several different fixing agents were used; Gilson's, Flemming's, Carnoy's, and Picro-acetic. The best results for the younger stages were obtained with a weak solution of Flemming. Fixation here was perfect with no cases of plasmolysis. In older stages, a strong solution of Flemming was used, diluted with twice its volume of distilled water. This gave excellent results where the outer coat had become cutinized. Infiltration ass obtained in all the younger stages by using $\mathrm{Xyl0l}$, but in the older stages this was not satisfactory. It was found after considerable experimenting that if the zygospores were allowed to stand in a weak solution of sodium hydroxide for $24-48$ hours, that the outer brown coat became softened and somewhat transparent. It was then a simple matter to dissect the zygospores from their thick coats and the 

suspensors. In other cases the material was carried through the killing and fixing process without dissection. In all cases after treatment with sodium hydroxide, the material was washed in running water for 12 hours in order to counteract any tendency to plasmolysis. They were then fixed at once in various reagents, here again the best results were obtained with a medium strong solution of Flemming. It was necessary to expose these to this solution for 48 hours as the coats are very slow of penetration. The usual grades of alcohol and cedar oil were then used, with an exposure of $8-7$ days in pure cedar oil. These were then carried through the grades of cedar oil and paraffin. The chloroform and xylol methods were also tried, but were not as satisfactory. All the younger stages were sectioned at 5 microns, while the older stages were sectioned varying from 7-30 microns, and studied in serial form.

In staining, Flemming's triple stain of safranin, gentian violet, and orange $G$, was used almost entirely, although Haidenhain's iron-haematoxylin method with Bismarck brown and saffranin as counter stains, was used in a great number of cases as a basis 

for comparison. These all proved highly satisfactory in their differential powers, and brought out the same structures in all cases.

LIFE HISTORY AND DEVELOPMENT.

As has been said, Sporodinia grandis readily produces

zygospores on a moist substratum. On the ends of certain aerial hyphae, there artses a slightly enlarged portion. Two branches come to stand opposite to eachother, and ultimately join.

Externally, there is no apparent difference between the two branches except that one appears smaller than the other in some few cases, but this seems to be the result of physiological rather than of morphological conditions. The protoplasm flows out into these enlarged ends, gradually becoming denser and denser, until the ends are finally closely filled, vacuoles being found only farther back in the filaments. Up to this point, the present investigations agree with Leger, Gruber, and Dangeard. Leger describes two types of branches at this point, one in which the protoplasm fills both of the sexual branches, and another in 

which the protoplasm is retracted from the wall. In the present studies, however, the protoplasm passes out to the wall in all cases but later retracts. In a very few cases, the protoplasm retracts in both branches, but even here the retraction is more evident in one than in the other, and in the majority of cases the retraction is evident in one side only. Contrary to the results obtained by Miss McCormick the author has been unable to distinguish any marked differential staining properties between the two adjacent gametangia. A granular substance usually fills the intervening space where the protoplasm has drawn back from the walls. If, as in some cases, the retraction is apparent for some distance down the lateral walls, then this area is also filled with this granular substance. This was particularly evident in preparations where irom-haematoxylin and Bismarck brown were used as stains. The granular substance always stains brown. This is probably the substance described by Vuillemin (14) which forms the secondary membrane before resorption takes place. The significance of the retraction will be considered later. Many nuclei are carried in with the protoplasm! and while 

in no cases, was nuclear division actually possible of demonstrat tion, yet there are many nuclei found in pairs, and there is a marked increase in the number which can hardly be explained on the basis of subsequent migrations. The nuclei are extremely small but when magnified 2000 times, they show the characteristic appearance.(Plate 1, figure 2a.) The main part of the nucleus is granular in appearance and stains violet or slightly red,depending on the length of exposure to the reagents. In the center of the nucleus, one finds a small intensely red-staining body that is entirely constant through all the stages of development. The constancy of this central body nould seem to suggest that it is chromatin in nature, rather than that it is a nucleolus, but as long as no nuclear divisions have been observed it remains a question. The nuclei are aggregated at the ends at this stage, only a few scatterdd ones occuring back in the more vacuolate parts. (Plate 1, figure 1,2, )

Vuillemin(14) and Dangeard (10) have offered evidence as to the fusion and the nature of the contiguous walls of the gametangia, and it will suffice here to say that these two walls 

coalesce, forming a single separating partition between the two structures. About this time and, in most cases, before the dissolution of the separating wall, large vacuoles are formed a short distance back in the swellings. These vacuoles seem to be constant except in a few cases where the cleavage furrow seems to cut in from the surface of the branches without the aid of the vacuoles. This is rare, however, and in the majority of cases the large wedge-shaped vacuoles are quite evident. (Plate l, figure 4) These cut in until only an isthmus of protoplasm remains as a connection between the vacuolar and the denser portions. In some cases, the turgidity of the filament seems to close the furrow but refusion never occurs. The line of cleavage remains distinct and very soon a new wall is built in from the outer walls. (Plate 1, figures 5,6.) Dangeard has suggested that this wall is in the form of a diaphragm which gradually closes in, but he offers no evidence to support this view. In the present preparations however, the relation of this is clearly shown. As the wall, which is in the form of a disk or diaphragm, closes in, the Infler edge of it becomes thickened, and when it ultimately 

comes together, the cellulose-like deposit continues to pile up for a short time, forming a papilla-like projection which extends out into the cut off portion, and is the "canal" described byleger. Vuillemin has failed to recognize this in his study of the membrane formation of the zygospores. In figare 6,Plate 1, the inner edge of the diaphragm is shown, as it closes in, and the narrow neck of protoplasm which remains. That some tension exists here is evident from the nature of the protoplasm at this point. The vacuoles and nuclei are elongated and lie parallel to the long axis of the isthmus. (Figure 6a) Further cleavage is caused by the inward cutting of the new membrane. Ultimately the wall closes in and the narrow neck of protoplasm is severed. In doing so there is sometimes left, at the center, a small point that ralways stains brilliantly. It seems probable from its reaction that it is some of the material that is never entirely differentiated. Thus, it can be seen that this simple little structure which has received the passing attemtion of many, and had various functions alloted to it, is an unimportant result of the delimiting of the gametangium. In no cases, have the orifices described by Leger and Dangeard, been found. 

As soon as this wall is completed, and indeed, in

numerous instances, at the time of the formation of the large

furrows, the wall separating the two gametangia is gradually

dissolved, and the contents of the gametangium which is filled

to the wall, pushes through; the contents of the other gametan-

gium often retracts. The masses retain their individuality for

a short time, and the regions of contact react differently to the stains. Ultimately, however, the line of contact is obliterated and it is impossible to distinguish any difference except that along the line of fusion there is a graater granularity than else where. The number of nuclei here is greater also. Gradually as the mixing of the protoplasm takes place, it passes from a dense to a slightly vacuolar nature. It is at this stage that nuclear fusions are evident, first along the line of fusion, and then gradually throughout the structure as it becomes vacuolated. Fusions have been observed at the portions next to the limiting membranes which separate the zygospore from the suspensors. The nuclei that fail to fuse, soon show signs of degeneration or disorganization. In the mycelium and in the gametangium before fusion, the nuclei are 

small and the outer membrane is granular and not clear cut. (Plate 1, figures $2,3,4$.$) In fusion, the nuclei present the characteristic$ appearance of fusing nuclei. They come to lie in pairs, the nuclear membranes disappear between them, and the two deep staining bodies, described previously, come to lie within a single membrane. Ultimately these two bodies fuse, and we find only a large central body. (Platell, figure7a.) The fusion nuclei are approximately twice the size of the original nuclei, and the number of nuclei appears less than that just prior to fusion. Dangeard describes the fusions as occuring as the protoplasm passes from the vacuolate to the reticulate condition, and also as after the formation of the new limiting wall, or endispore. In no cases have fusions been observed as late as this in the present material, but always occurs prior to the formation of the warty wall, and during the vacuolate stage. In omly a few rare instances, have fusions been observed after the protoplasm has passed into the reticulate condition.

This fusion of nuclei resulte in the presence of tho sizes of nuclei at this time, that is, at the beginning of the 

reticulate appearance. The protoplasm assumes a thready appearance, and throughout the zygospore there is evidence of a nuclear disorganization which is restricted to the smaller nucle1. (Plate 1, fig.7.) At one time, the majority of these nuclei that are undergoing degeneration appear in a zone around the periphery, while those towards the center retaim their normal form. (Plate 2, figs. $8,8 a, 8 b, 9$.$) In degeneration the outer membrane remains$ distinct for some time, but the inner portion loses its granular appearance, and the central red-staining body increases in size and stains less densely. The whole nucleus appears eventually as a large red-dtaining mass. (Plate 2 , fig. $\not a$ ) The nuclei present the same appearance as they degenerate following the cutting off of the terminal cells.

About this time, there appear numerous spherical bodies of cytoplasmic origin. That these are not products of the disorganizing muclei seems evident from their early structure. At first they stain lightly violet or orange with triple stain. They are generally globular in shape although they vary somewhat. In tbe earlier stages, they are reticulate throughout, and are quite definite as individual bodies. Many originate near the margin 

of the zygospore, and others if they originate here, migrate in.

They appear to originate throughout the cytoplasm. The third wall is laid down by this time, and the zygospore has a clear, thick, very lightly staining, warty coat, outside of which is the brown warty coat. In some rare cases, the original outer membrane of the gametangia still persists. (Plate 2, fig.9,20)

The cytoplasm is thready in nature and has numerous vacuoles. The new cytoplasmic bodies are confusing at first, and only careful study of their function and fate has placed them as definite cell structures. They have,undoubtedly, been the cause of the confusion that has existed so long in this group. The structures described by Leger under the name of "spheres embryogenes" are most certainly identical with these structures. Dangeard described them as chromatic corpuscles but did not follow them very far, and arives at no conclusions as to their functions, aside from comparing them to the coenocentra of Stevens.

That these bodies are not nuclei is evident in a close study of their structure, and is more forcibly emphasized by their subsequent behavior. They gradually increase in number (Plate 3, fig.11.), as many as forty having been counted in one section of 

a zygospore. They increase in size and fusions occur between them without any reference to size or number. These irregularities of fusions would make any comparison between these bodies and nuclei, highly questionable. As they enlarge they show more definite structure. They have a dense reticulate central portion which stains a deep violet, so deep that in thick sections it is almost impossible to make out any dearils of structure (Plate $2, \mathrm{fig.lOb.}$ ); in thinner sections they are definite and quite uniform. Towards their margins, they gradually lose their dense structure and become loosely reticulate, appearing sponge-like. In no instances, under various stains and in serial sections, could anything that resembled a nucleolus or chromatin material, be discerned. In no cases, could nuclei be found within these bodies, but in all cases the nuclei already described(Plate2, fig 10a.) occured in meshes between these structures and the vacuoles. These structures, which will be refered to as oil plastids from now on, continue to enlarge and fuse. Through tests with osmic acid and chloroform, it is found that, from their first appearance, these plastid=like bodies are directly associated with the formation of oil. 

Aggregated at one side and irregularly distributed through the rest of the zygospore, appears an irregulat thready mass of an intensely red-staining material. That this is the result of the disorganization of nuclei seems unquestionable. In some cases, it has been possible to recognize the individual degenerating nuclei at this stage while others show their gradual coalescence. The ultimate fate of this material has not been determined as, so far, it has not been possible to carry investigations to the place of germination of the zygospores. That this material goes towards the nourishment of the zygospore in germination seems highly probable. This condition was observed in material as young as eight weeks, and still persisted as late as twelve weeks. Thus it would seem that few, if any, changes occur after this before germination. The oil plastids described may be related to the "elaioplasts" described by Zimmerman (15) and Racioborski(16) as occuring in various leaves. Somewhat similar bodies are described by Barker (17) as having to do with the formation of glycogen in yeasts. As early as six weeks, the oil plastids may have fused together to form one or twd large bodies (Plate 3, figs. 13,14,15) 

In some instances three of these bodies are present. (Plate 3, fig.13)

In fresh material they appear as large oil globules, due to the

fact that they are completely saturated with oil to the point

that if slightly pressed, the oil exudes in a large globule which is immediately soluable in chloroform. This material is not very refractive and is amer colored. It assumes the shape of the plastid, not always being in the form of a definite rounded globule.(Plate 4, figs. $21,22,23,24,25,26$. )

These plastids are undoubtedly the "embryo spheres" of Leger, the nuclei of Lendner, and possibly the nuclei described by Miss McCormick. If one has not traced them through all the earlier stage in a careful and painstaking manner, their extreme prominence in the cell would lead one to ascribe a more significant role to them. Leger found two of these spheres present in all cases, and maintains that they fuse upon germination. But in the material at hand, these bodies have been found in various stages in material from 8-12 weeks old. (Plate 3 , figs.14,15.) In the greater majority of instances, a single plastid appears, while in the rest two or three occur at an age of three months, and small plastids may be observed at this time also; The ultimate fusion of 

these large plastids, is doubtful.

Dangeard describes in older zygospores, the presence of 10-20 corpuscles which he suggests are the results of reorganized mucorine crystals which have merely incresed in number and lost their crystalline form.

According to Miss McCormick, the large structure that she finds associated with the nucleus has been identified by Stevens as similar to the coenocentra desbribed by him in Albugo. Miss McCormick, however, describes them as arising at the point of contact of the suspensors before the gametangia are cut off. Stemens (18) in Albugo Bliti regards this body as an "organizer" while Wager (19) regards it as having to do with the behavior of the sexual nuclei. It is described as occuring prior to fertilization. In no cases in the present investigations, were these structures observed before nuclear fusions took place. The only possible similarity to any such structure as described by Miss McCormick as originating before the gametangia are cut off, id the dense aggregation of the protoplasm at the points of contact, which is unrelated to the formation of the oil plastids.

The fate of the nuclei through these varying changes is 

important and significant. Considerable difficulty was encountered at this point. The nuclei do not stain as brilliantly, and owing to the brittle wall of the zygospore, it was necessary to section the material at $14-30 \mu$ so that the nuclei did not stand out as clearlyas in the earlier sections. It was found, however, that the nuclei persist in almost the same form and structure as at the time of fusion. (Plate 3, fig. 15.) In the oldest stages obtained, the nuclei show a somewhat greater affinity for the daffranin, and in many cases the whole karyoplasm stains red, but retains its granular appearance, and in no resembles the degenerating nuclei. In the earlier stages, the nuclei are scattered through the cytoplasm but lie close to the oil plastids. At the time of the appearance of the single large plastid, the nuclei can be found in close proximity to the surface of the plastid, away from the regions of nuclear degenerations. The oil is undoubtedly a reserve substance. The protoplasm is in a thin parietal layer at this stage. This condition persists throughout all the older material secured, and it seems highly probable that no further changes occur until germination. 

Further investigations have been delayed, but it is the object of the author to determine next, the fate of the nuclei, oil plastids and the disorganized nuclei when germination takes place.

From Leger's work and observation it would seem that the zygospore in germination uses up this oil reserve; the plastid is probably disorganized; and the nuclei undergo division, forming the nuclei of the next vegetative generation. This is, however, purely hypothetical, and is in no way offered as observations and conclusions of the author.

THEORETICAL CONSIDERATIONS.

The significance of several of the above described phenomena seems worthy of further consideration. In the first place, the retraction of the protoplasm at the point of contact of the two branches, is constant enough to appear significant. It is only in rare cases that the protoplasm retracts in both branches. Vuellimin (140 has described the retraction as making more evident the formation of the secondary membrane. He has, furthermore, described the laying down of this wall as occuring first on one side and then on 

the other, in some cases. That this constant retraction has more significance than merely as a result of the formation of this temporary membrane, seems probable. When the membrane is dissolved, the gamete that has been closely applied to the partition, pushes through. In most instances, a decided current of the protoplasm, is evident. The other gamete generally shows a retraction before the force of the inflow. Therefore, it would seem that one gamete was more or less passive in this process, while the other exhibited a certain constant activity. Further differentiation of the gametes was not apparent, either as to staining reaction or the number and size of the nuclei.

The term gametangium has been used to designate the end of the branch that is cut off by the cross wall. The term gamete has been applied to the multi-nucleate mass that is included within the gametangium.

If we consider the term gamete as synonymous with the terms cell or protoplast, then we must define the term cell to justify this terminology, as there is much disagreement on this point. The definition of a cell as first given by Leydig (20) in 1857, and Max Schultze (21) in 1861, is, "A cell is a mass of 

protoplasm containing a nucleus." ---" in which both nucleus and protoplasm arise through the division of the corresponding elements of a pre-existing cell." This definition has been reduced to mean, by many at least, that a cell is a nucleus with a certain amount of associated protoplasm. Sachs (22) has suggested the term 'energid' to represent'the nucleus with that portion of the active protoplasm that falls within its sphere of influence, the two forming an organic unit, both in a morphological as well as in a physiofogical sense.'

Pfeffer (23) define each protoplast as a cell and includes the Siphoneae or other malti-nucleate protoplastsas unicellular plants. With regard to the term'energid', he says, "It seems hardly necessary to introduce the term energid to represent the combination of a nucleus and cytoplasm which forms the living cell unit. Sachs sees to state that in multi-nucleate cells, each energid is to be regarded as a definite whole, even though $\not 6$ no visible line of demarcation exists. It is, however, hardly credible that multi-nucleate cell is made up of a series of energids, in each of which the living substance remains separate and distinct from its neighbors, and indeed, it may be dieectly ge 

seen that the cytoplasm in the neighborhood of each nucleus is continualy changing where streaming takes place; while if the nucleus rotates also, it is being continually associated with new portions of the non-moving ectoplasm layer. Moreover, it is quite certain that the protoplasm lying between a number of nuclei is influebced by all of them to an extent corresponding to their respective distances from it, so that all these considerations compel us to regard a multi-nucleate cell as a single unit, both morphologically and physiologically."

Ewart (24) in criticism of Pfeffer's views, says," The consideration of a question, essentially morphological, from a purely physiological point of view, leads to confusion...- In the widest possible sense any differentiated mass of protoplasm, with or without a cell wall, with one or many nuclel, will constitute a cell, while a protoplast is formed by the symbiotic union of a single nucleus and a single plasma mass only, and represents the lowest unit capable of soparate existence, A number of protoplasts may be united toggther to form a multi-nucleate coenocyte." Ewart, thus, differentiates between the cell and a protoplast, and with very little justification. A protoplast from this 

standpoint would not be a physiological unit in any sense. And even as this oannot be decided from a purely physiological point of view, neither is Ewart justified in concluding that the individual nuclei in a coenocyte, are protoplasts from a purely morphological point of view. In Sporodinia grandis, it is highly questionable if this would hold true morphologically. In PIate 1, figure 3 , it will be seen that the nuclei of the mycelium do not have any defintte area of cytoplasm around them. In many cases, the nuclei are aggregated. It seems absolutely impossible to sonsider any one of these nuclei as a morphological or a physiological unit when considered from the standpoint of its individuality as a protoplast. At the time of fusion the nuclei are circulating through the cytoplasm, and when two nuclei are brought together it is the nuclei that fuse without any reference to the surrounding cytoplasm in so far as can be determined. A further point in the cobsideration of the structural unit here, is that at no time in the life history of this fungus does a uni-nucleate stage occur. As Harper (25) has shown and as the author has observed, even at the time of spore formation, the masses of sporesthat are cut out are never uni-nucleate but always multinucleate. According to 

Ewart's definition of a protoplast then, we must consider the spores as a complex of cells or protoplasts. Does this seem justified when we consider that from very other standpoint except that the mass has several nuclei instead of one, it is in all senses an entity, and responds, functions, and is structurally, a protoplast?

Harper (26) discusses these points at some length. He refers th the red algae. Here instances are found where regularly uni-nucleated cells and multi-nucleated cells occur in the same thallus. Under the consideration that a nucleus and its associated cytoplasm represents the unit of structure, we must call the structure that contains a single nucleus a cell, and the other that contains several, a complex of cells.

Harper defines the cell: "Anatomically and morpholog cally the cell is a mass of protoplasm enclosed by a continuous, semi-permeable, irritable membrane, and from this standpoint it may be one or many nucleated. That there is a certain unity in a mass of tissue such as a palisade parenchyma macie up of cells combined in a leaf for the performance of a definite function, is not to be questioned, but this unity is achleved by the 

combination of the activities of a number of co-ordinate individuals. Each cell in the tissue receives its own nutrition and throws off its own wastes, and each receives and reacts to stimuli from its environment.-.-. The continuous plasma-membrane enclosing a coenocyte is plainly in relation to the other cell contents, to be compared to the same structure in a uni-nucleated cell, rather than with the aggregate of membranes which bound off a mass of tissue from its environment, and the cells of the tissue from each other."

"The sum of reactions in a mass of tissue-cells may be in harmony and serve some common end for the organism, but they are not the less independent in their origin and in their accomplishment in that they are co-ordinated."

As Ewart suggests, it is not possible to decide on the nature of a protoplast or a cell as an entity from a purely physiological point of view. On the other hand, it is not possible to isolate a structure as a morphological unit regardless of its physiological individuality. In Sporodinia, therefore, since the nuclei do not exhibit a morphological individuality at any time 

or in any respect, and since the whole contents of the gametangium does exhibit a unity, both morphologically and physiologically, it has seemed justifiable to speak of the multi-nucleate mass as a gamete and not as a coeno-gamete, as Stevens has termed the. multi-nucleate oosphere of Albugo Bliti. Dangeard has choseb to consider the individual nuclei as "semal energios" or gametes, thus maing the whole structure a complex of gametes or a coenogamete. But as he has pointed out, the nuclei exhibit no individuality aside from their fusions, and there is certainly no differentiation into regions or units that can be considered protoplasts.

On the other hand, the whole gamete reacts as a unit, as has been shown. One gamete shows a greater activity than the other, the individual nuclei being only passive structures as far as can be determined. The cytoplasm fuses as does the cytoplasm of the individual gametes of a uni-nucleated structure, as in the case of Spirogyra. It mixes gradually and the nuclei fuse in pairs, several hundred instead of two. The ultimate product is a single many nucleated protoplast, which responds and functions as a unit at all times. Futthermore the cell inclusions, oil 

plastids, and mucorine crystals, exhibit no relation to the nuclei as individual protoplasts or energids, but bear the same relation to the whole gamete or protoplast as do the chloroplasts or crystals of a uni-nucleated cell.

RESEMBLANCE OF COTNOCENTRA AND OIL PLASTIDS.

That the coenocentra of Wager(19) and Stevens (18) are identical in nature and function with the structures described as oil plastids in the present paper, seems probable. Nager describes these structures in Albugo candida as having to do with the behavior of the sexual nuclei. Stevens in Albugo Bliti is inclined to attribute to them the function of "organizers". They appear with certain dynamic changes and zomation in the celd. Further on, Stevens describes a certain form of oil which is formed in globules, of a clear honey-yellow color. These originate near the wall of the oospore, enlarge, fuse, and form several large globules. "This results finally in a condition where the entire central region is occupied by a curious irregular globular structure, which stains much as the oils have earlier, but which 

is certainly not of a fluid consistency. It is characteristic of $\mathrm{b}$ the winter spore."

As will be seen at once, this structure is undoubtedly identical with that described in the mature zygospore of Sporodinia grandis. It is further highly probable that it originates in the same way. Sterens points out a close similarity between the staining reaction of this large oil globule and the reaction of the coenocentrum. It would eem then that the coenocentrum is merely an early stage in the formation of the oil plastids and results from the fusion of many small plastids. In Sporodinia, the plastids do not appear as early as described in Albugc. 

SUMMARY •

1. In the early stages of copulation, there is no difference morphologically between the two sexual branches. Later, there is a characteristic and constant retraction of the protoplasm in one of the gametangia.

2, The nuclei are small and show the same structure, sizem and staining reaction, as those of the mycelium.

3. Vacuoles and cleavage furrows are formed. A new membrane is formed by the ingrowth from the lateral walls. A central thickened portion is formed.

4. The wall betweem the gametangia is dissolved, and the gametes mix, one always flowing into the other.

5. Nuclear fusions occur progressively as the protoplasmic masses mix.

6. Two sizes of nuclei result.

a. Large nuclei, the results of fusions.

b. Small nuclei, of a simple nature which fail to fuse.

7. The formation of the second wall takes place, and the unfused nuclei,as well as those in the suspensors, show degeneration. 8. Mucorine crystals are also present through all the earlier 

$-40-$

stages.

9, There next appears globular reticulate cytoplasmic structures,

Oil is associated with these from the first. They fuse irregularry and produce ultimately one or two large bodies which are saturated with oil. This oil reacts to the osmic acid test, and is soluble in chloroform.

10, Disorganized nuclei appear massed on one sickie and scattered less densely over the remaining potion. Larger fusion nuclei which have retained their characteristic form and react to triple stain in the same way, are found around the periphery of the plastids. The protoplasm is much reduced and forms only a thin parietal layer.

11. This state of affairs persists until germination.

Department of Botany,

University of Missouri.

approved may 15,1912

George in. Reed 



\section{BIBLIOGRAPHY •}

1. Ehrenberg, C.F. 1820. Verhandlung. der Gesellsch. nat. Freund. Berlin. Bd.l.

2. De Bary, A. 1864. Beitrage zur Morphologie und Physiologie der Pilze. Syzygites megalocarpus. pp.14-88. Taf. 5-6.

3. Brefeld, 0. 1872. Botanischen Untersuchungen uber Schimmelpilze (Mucor, Chaetocladium, Piptocephalis.) Leipsig, 1872.

4. Van Tieghem, et Le Monnier. 18\%3. Recherches sur les Mucorinees. Annales des Sciences naturelles, 5e serie, t.17, 1873.

5. Dangeard et Leger.1894. Recherches sur la Structure des Mucorinees Compt. Rend. CXV111, 1894, p. 430. La Reproduction Sexuelle des Mucorinees. Compt. Rend. Mars 5, 1894, p. 54r-549.

6. Leger, M. 1895. Structure et Developpment de la Zygospore du Spododinia grandis. Revue Gen. de Bot., 1895. p. 481-486.

7. Gruber, E. 1901. Uber das Verhalten der Zellkorne in den Zygosporen von Sporodinia grandis. Link.

Ber. d.d. Gesell. 1901. p.51-55.

8. Mottier, B. M. 1904. Fecundation in Plants. Carnegie Instit. 1904

9. Dangeard,P.A. 1906. La Fecondation nucleaire chez les 

Mucorinees.

Compt. Rend. 1906. p. 645-646.

10. Dangeard, 1906. Les Mucorinees.

Le Botaniste, 9e serie, 1906,p 227-253.

11. Lendner, A. 1908. Recherches Histologiques sur les Zygospores

du Sporodinia grandis.

Herb. Boissier. 2e, 1908, p.77.

12. Moreau, I. '1911. Fusions de Noyaux et degenerescence nucleaire dans la Zygospore.

Bull. Societe Mycol. de France. 27: 1911, p. 334-341.

13. McCormick, F. 1912. Development of the Zygospores of Rhizopus nigricans.

Boatnical Gazette. vol.Ll11, no.1, 1912,p. 61.

14. Vuillemin,P. 1903. Importance Taxomomique de l'appareil

Zygospore des Mucorinees.

Bull. Soc. Myc.Fr. t.19, 1903,p. 106-108.

Evolution des quatre assises protectrices de la zygospore chez quelques especes de Mucoracees.

Annales Mycologici von Sydow. 2: 1904,p. $48 \%$.

15. Zimmerman, A. Elaioplasten. 

Beitr. Morph. Physiol. d. Pflanzenzelle, Heft 3, p. 185.

16. Racioborski,M. 1894. Elaioplasten bei Liliaceen.

Bot, Centralblatt. 57: 1894,p.305-307.

17. Barker,B.T.P. A Conjugating Yeast.

Ph11. Trans. Roy. Soc.B. 1901, p. $467-485$.

18. Stevens, F.L. 1899. The Compound Oosphere of Albugo Bliti.

Botanical Gazette, vol.XXV111, no. 3-4, p. 149-176, 225-246.

19. Wager,H. 1896. On the Structure and Reproduction in Cystopus candidus.

Ann. Bot., 10: 1896, p. 295-341.

20. Leydig, Lehrbuch der Histologie, 1857, p. 9 .

21. Schultze, M. 1861. Arch. Anat. u. Phys. 1861, p. 11.

22. Sachs, Flora 92, p. 57.

23. Pfeffer, T. Physiology of Plants, vol.1.p.60.

24. Ewart, A.J. Pfeffer's Physiology of Plants. p.61.

25. Harper, R. A. Cell Division in the Sporangia and Asci. Ann. of Bot. vol.13, 1899, p.467-525.

26. Harper, Sexual Reproduction in Pyronema confluens and the Morphology of the Ascocarp. Ann. of Bot. 1900, p. 375-382. 

(All figures were drawn with the camera lucida and with the Zeiss apochromatic objectives and compensating oculars. Figs. $1,2,4,5,7,8,9,10$, and $11-15$, are drawn with objective $16.0 \mathrm{~mm}$. ocular 12. Figs. 3, 2a, 4a, 6a, 7a,8a,8b, 10a and 10b, were drawn with obj.1.5 mm. and oc.8. Fig.6 is drawn with obj. $4.0 \mathrm{~mm}$. and oc. 4. ) All figures are reduced one half.

Figure 1. Young branches at copulation.

Figure 2. Branches showing aggregation of protoplasm at the ends and characteristic retraction of one mass.

Figure 3. Section of mycelium showing corm and size of nuclei.

Figure 4. Gametangia with middle wall dissolved, and the format tion of vacuoles and furrows.

Figure 4a. Nuclei and mucorine crystals.

Figure Portion of suspensor and gametangium with new wall formøng.

Figure 6. Same as fig. 5 , only in greater detail to show the thickening of the edge of the diaphragm and its relation to the neck of protoplasm. 

Figure 6a. Nuclei from fig. 6 , to show elongation in plane of is thmus.

Figure 7, 7a. Zygospore after limiting walls have been formed and at the time of nuclear fusions. 7a. gives various stages of fusion nof nuclei and indicates the difference in size of the nuclei before and after fusions.

Figures $8,8 \mathrm{a}$, and $8 \mathrm{~b}$. The zygospore at the reticulate stage, nuclear degeneration and zonation. $8 a$, shows nuclei in process of degeneration. 8b. typical fusion nuclei.

Figure 9. Zygospore in which zonation of disintegrating nuclei is more marked.

Figure 10. Zygospore after formation of endospore and at the time of the appearance of the oil plastids. 10a, Nuclei.

Figure 11,12, Increase is size and number of the oil plastids. 11 under triple stain and fig,l2 under iwon haematoxylin. Figures 13, 14. Formation of several large plastids.

Figure 15. Mature zygospore containing one large and several small plastids. 15a Nuclei from mature zygospore. 15b A section from the large oil plastid showing its reticulate nature. 

Figures 16- 2.1, Zygospores in fresh condition showing the characteristic oil globules in various stages. 

PlateI.

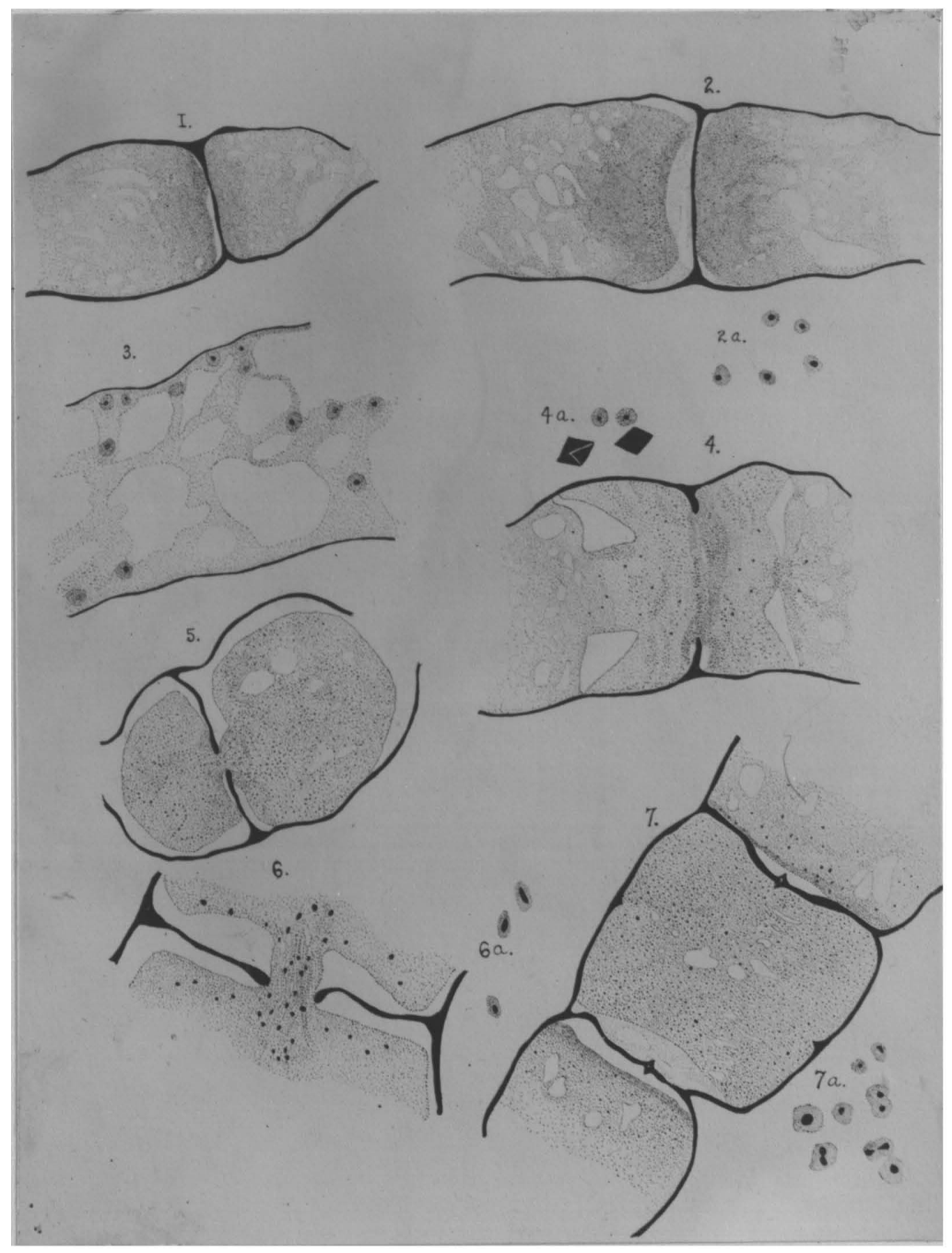



Plate II.

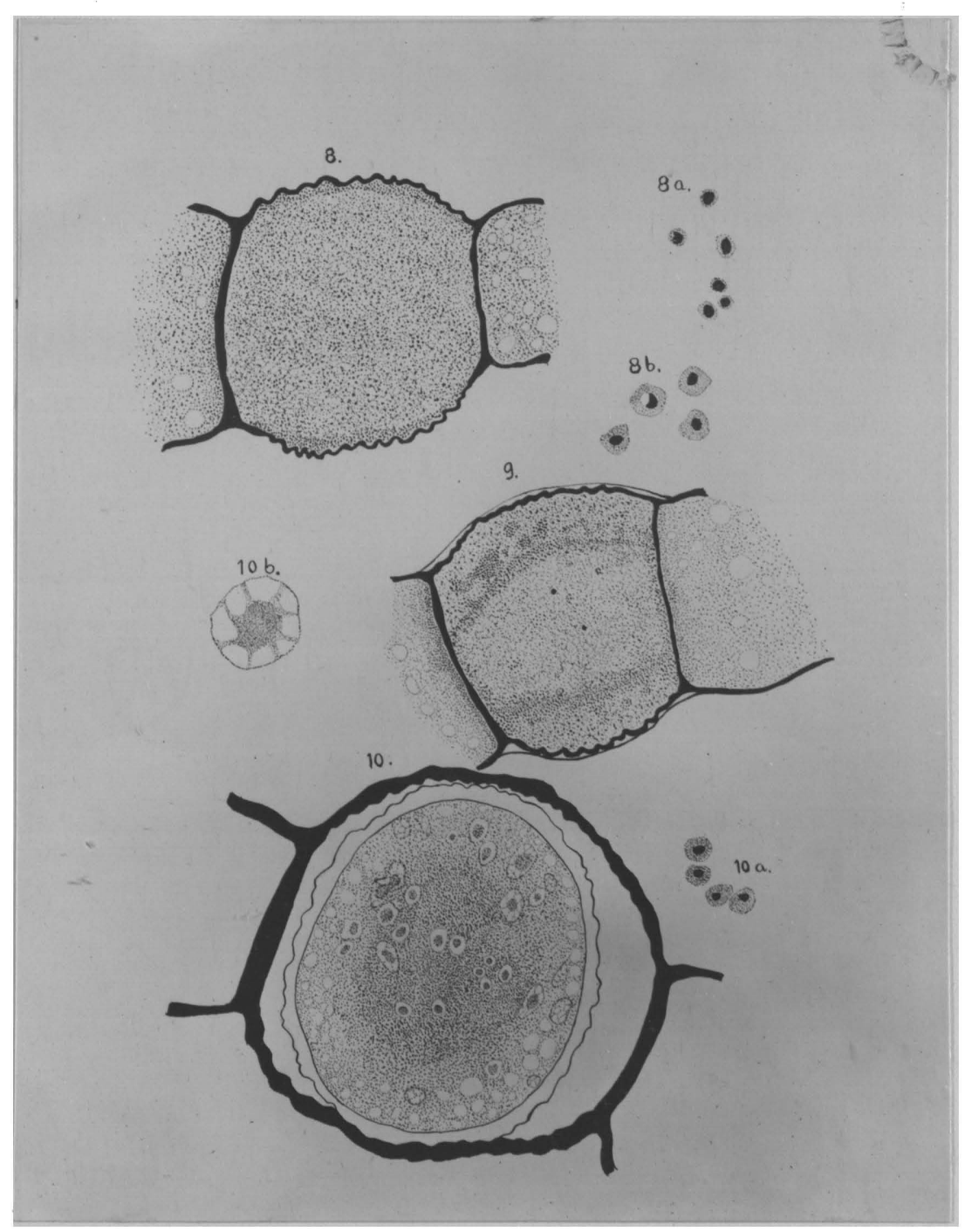



Plate III.

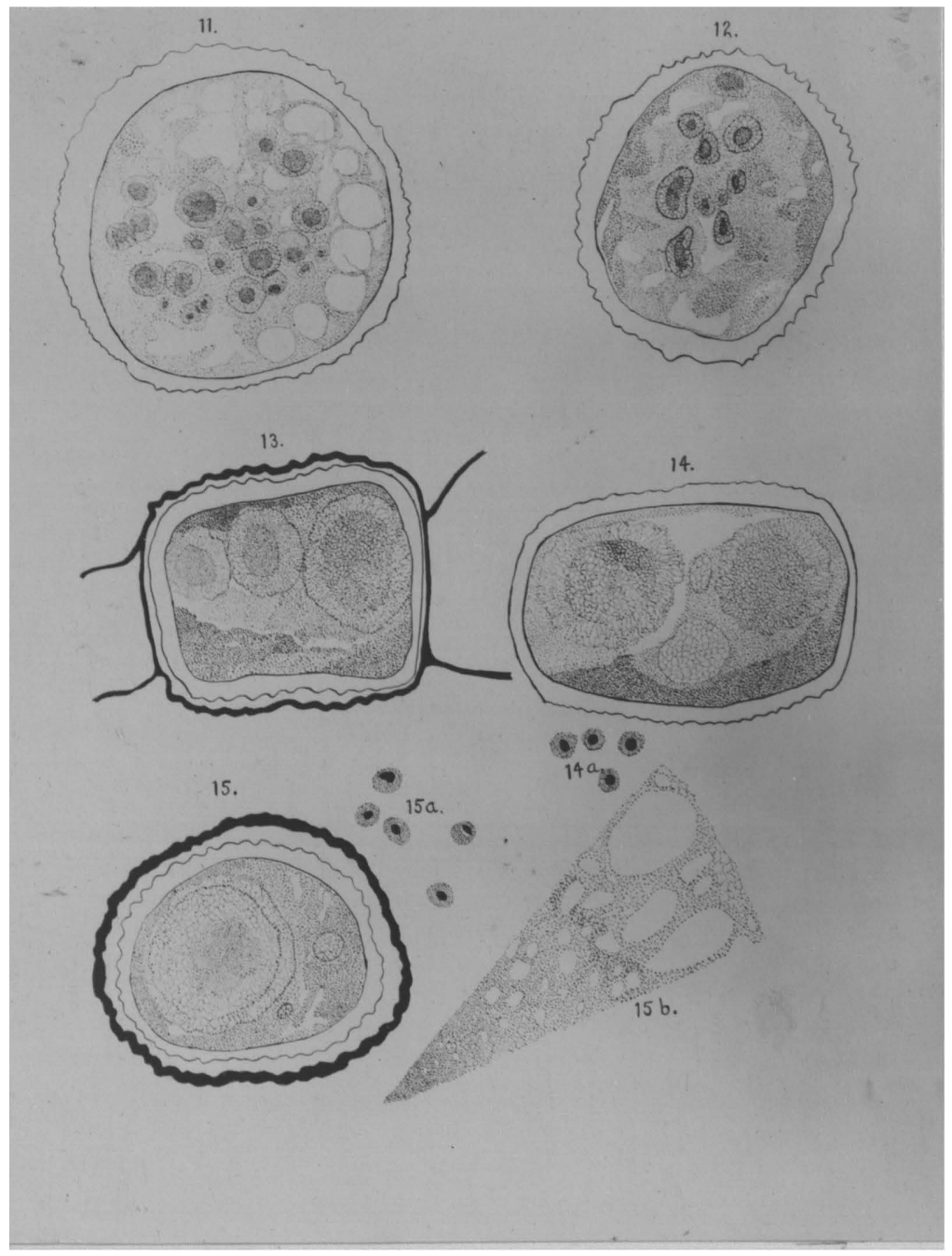



Plate II.

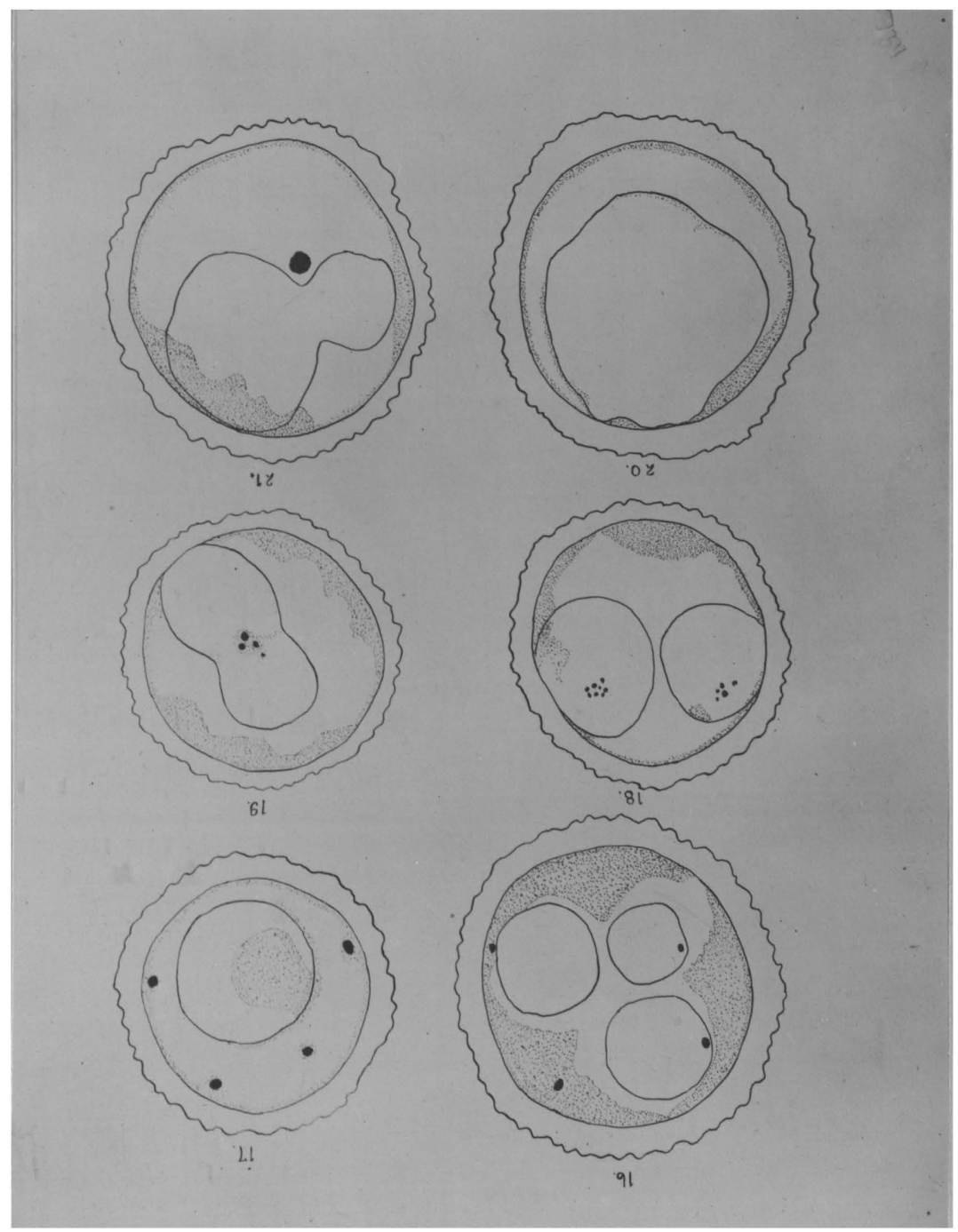







\section{$378.7 M 71$ $X K 25$}
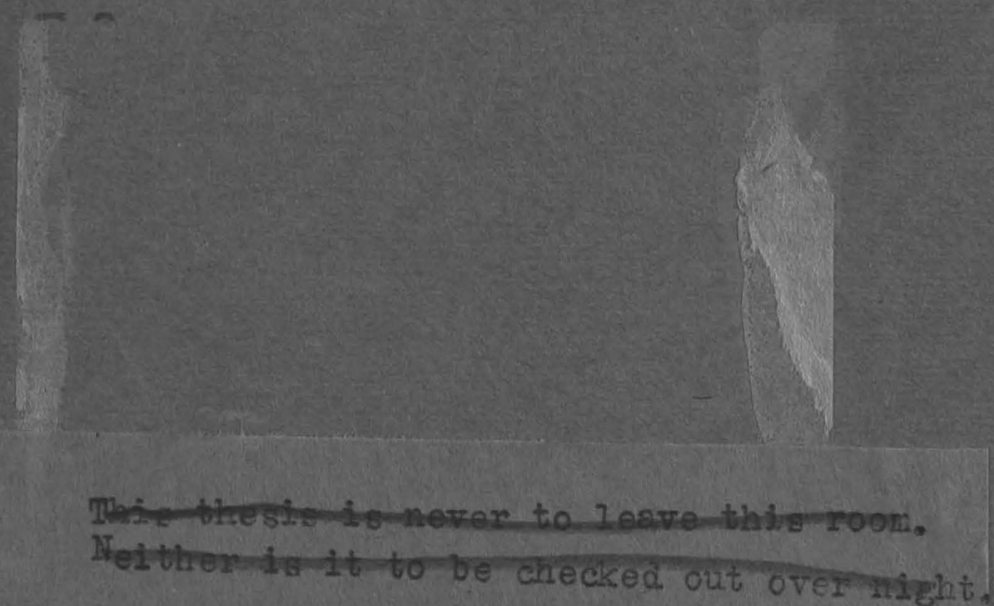
